\title{
18.
}

\section{Eine Bemerkung zum Pascalschen Theorem.}

(Von.Herrn Otto Hesse, Professor an der Universität zu Königsberg.)

In seinem grofsen Werke: „Systematische Entwickelungen etc. 1832." stellt Steiner (S. 311) folgende Theoreme zusammen:

1. „Irgend sechs Puncte eines beliebigen Kegelschnitts bestimmen 60 ein"geschriebene einfache Sechsecke."

2. „In jedem der letzteren liegen die drei Puncte, in welchen die gegen„überliegenden Seiten sich schneiden, in einer Geraden $\boldsymbol{G}$; so dafs also "60 solcher Geraden $G$ Statt finden."

3. „Von diesen 60 Geraden gehen drei und drei durch irgend einen "Punct $\boldsymbol{P}$; so dafs also 20 solcher Puncte $\boldsymbol{P}$ entstehen."

4. Und von diesen 20. Puncten $P$ liegen 15 mal 4 in einer Geraden $g$; "so dafs jeder in drei solchen Geraden liegt."

Steiner fügt hierzu noch die Frage:

„Welche Beziehungen haben diese 15 Geraden $g$ weiter zu einander?"

Von diesen Sătzen ist derjenige No. 4., wie Plücker in der Note zum Pascalschen Theorem im 34ten Bande dieses Journals ausdrücklich bemerkt, aus seiner Abhandlung vom Jahre 1829 (in diesem Journal Bd. 5. S. 268) in das Steinersche Werk übergegangen. Es ist daher anzunehmen, dafs Steiner eine andere Erledigung seiner Frage verlangt, als die erwähnte frühere $A b-$ handlung von Plücker gewährt. Weder die Note von Plücker, noch die andern in diesem Journal enthaltenen Schriften, welche das Pascalsche Theorem wieder in Erinnerung bringen, berühren die interessante Steinersche Frage. Ich nehme Gelegenheit, dieselbe durch Anführung einiger aus meinen Universitätsvorträgen entnommenen Sätze zu beantworten.

5. „Die 15 Geraden $g$ entsprechen den 15 Systemen von drei Geraden, „welche sich durch die sechs Puncte des Kegelschnitts legen lassen, in „folgender Art:

,Wenn $u=0, v=0, w=0$ die Gleichungen von drei Geraden „bedeuten, welche durch die sechs Puncte des Kegelschnitts hin- 
,durchgehen, dessen Gleichung sich bekanntlich auf die Form

$$
\boldsymbol{A} u^{2}+B v^{2}+C w^{2}+2 F v w+2 G u u+2 H u v=0
$$

,zurückführen läfst, so ist:

$$
\boldsymbol{A F u}+\boldsymbol{B G} \boldsymbol{v}+\boldsymbol{C H} \boldsymbol{w}=\mathbf{0}
$$

"die Gleichung der Geraden $g$, welche dem Systeme von drei Ge,raden $u=0, v=0, v=0$ zugehört."

Die drei Geraden $g$, welche auf diese Weise den drei geraden Seiten eines der 60 Sechsecke, den drei ungeraden Seiten desselben Sechsecks und den drei Diagonalen entsprechen, welche die gegenüberliegenden Ecken des Sechsecks verbinden, schneiden sich in einem und demselben Puncte $\boldsymbol{P}$.

Eben so schneiden sich die drei Geraden $g$, welche dem ersten Paare der gegenüberliegenden Seiten des betrachteten Sechsecks und der ersten Diagonale (welche das letzte Eckenpaar des Sechsecks verbindet), dem zweiten Paare gegenüberliegender Seiten und der zweiten Diagonale, dem dritten Paare gegenüberliegender Seiten und der dritten Diagonale entsprechen, in einem und demselben Puncte $p$, welcher in Rücksicht auf den Kegelschnitt der harmonische Pol zu dem vorhin gedachten Puncte $\boldsymbol{P}$ ist.

6. „Wenn die Ecken von drei Dreiecken auf drei Geraden $g$ liegen, welche ,s sich in einem und demselben Puncte $\boldsymbol{P}$ schneiden, so schneiden sich „,die entsprechenden Seiten je zweier von diesen Dreiecken in drei „P Puncten, welche auf einer Geraden $\gamma$ liegen, und die drei Geraden $\gamma$ ,schneiden sich wieder in einem und demselben Puncte $p$.

In der That: wenn man durch $a=0, b=0, c=0$ die Gleichungen der Seiten des ersten Dreiecks, durch $a^{\prime}=0, b^{\prime}=0, c^{\prime}=0$ und $a^{\prime \prime}=0$, $b^{\prime \prime}=0, c^{\prime \prime}=0$ die Gleichungen der Seiten des zweiten und dritten Dreiecks, endlich durch $g=0, g^{\prime}=0, g^{\prime \prime}=0$ die Gleichungen der drei Geraden $g$ bezeichnet, auf welchen die Ecken der drei Dreiecke liegen und welche sich in dem Puncte $\boldsymbol{P}$ schneiden, so lassen sich die 12 willkürlichen Constanten, welche die genannten Gleichungen als Factoren enthalten, unter den aufgestellten Bedingungen so bestimmen, dafs folgendem Systeme von Gleichungen identisch genügt wird:

$$
\begin{aligned}
& b-c=b^{\prime}-c^{\prime}=b^{\prime \prime}-c^{\prime \prime}=g, \\
& c-a=c^{\prime}-a^{\prime}=c^{\prime \prime}-a^{\prime \prime}=g^{\prime}, \\
& a-b=a^{\prime}-b^{\prime}=a^{\prime \prime}-b^{\prime \prime}=g^{\prime \prime} ;
\end{aligned}
$$

und umgekehrt, wenn diesem Systeme identischer Gleichungen Genüge ge- 
schieht, so stellen obige Gleichungen die Seiten von drei Dreiecken dar, deren Ecken auf den drei Geraden $g$ liegen, welche sich in einem und demselben Puncte schneiden.

Bezeichnet man nun die Ausdrücke $a^{\prime}-a^{\prime \prime}, a^{\prime \prime}-a, a-a^{\prime}$ respective durch $\gamma, \gamma^{\prime}, \gamma^{\prime \prime}$, so folgt aus dem aufgestellten Systeme identischer Gleichungen sogleich folgendes System:

$$
\begin{gathered}
a^{\prime}-a^{\prime \prime}=b^{\prime}-b^{\prime \prime}=c^{\prime}-c^{\prime \prime}=\gamma, \\
a^{\prime \prime}-a=b^{\prime \prime}-b=c^{\prime \prime}-c=\gamma^{\prime}, \\
a-a^{\prime}=b-b^{\prime}=c-c^{\prime}=\gamma^{\prime \prime},
\end{gathered}
$$

dessen geometrische Deutung den angeführten Satz giebt.

Ich führe diesen Satz, dessen erster Theil bekannt ist, hier an, weil er ein Bild von der Lage der 20 Puncte $\boldsymbol{P}$ und der 15 Geraden $g$ zu einander giebt. Die Puncte $\boldsymbol{P}$ nemlich werden- durch die 9 Ecken der drei Dreiecke, durch die 9 Schnittpuncte der entsprechenden Seiten je zweier vón diesen Dreiecken, und durch die Puncte $P$ und $p$ repräsentirt; ferner die 15 Geraden $g$ durch die 9 Seiten der drei Dreiecke, durch die drei Geraden $g$, auf welchen ihre Ecken liegen, und durch die drei Geraden $\gamma$.

Die durch die 20 Puncte $P$ und die 15 Geraden $g$ gebildete Figur ist demnach symmetrisch: in der Art, dafs man, wie von dem Puncte $\boldsymbol{P}$ ausgehend, zu dem Puncte $p$ gelangt, eben so in derselben Figur von dem Puncte $\boldsymbol{p}$ ausgehend zu dem Puncte $\boldsymbol{P}$ gelangen wird. Dasselbe gilt von allen 20 Puncten $\boldsymbol{P}$. Auf diese Weise paaren sich die 20 Puncte $\boldsymbol{P}$, und diese Punctenpaare stehen wieder zu dem Kegelschnitt in der Beziehung, welche der folgende Satz angiebt:

7. „Die 20 Puncte $p$ bilden 10 harmonische Polenpaare des Kegelschnitts." Den aus diesem Satze durch das Princip der Reciprocität abgeleiteten Satz habe ich am Ende 'meiner Schrift ,Über das geradlinige Sechseck auf dem Hyperboloïd" bewiesen (S. dieses Journal Bd.24.).

Königsberg im September 1849. 